\title{
Estructura de las redes de colaboración científica entre las universidades españolas
}

The structure of scientific collaboration networks between spanish universities

\author{
Carlos Olmeda Gómez, Antonio Perianes-Rodríguez y María Antonia Ovalle-Perandones \\ Universidad Carlos III de Madrid. Grupo SCImago. Departamento de Biblioteconomía y Documentación, \\ cl Madrid 128, 28903, Getafe. Madrid, carlos.olmeda@uc3m.es
}

\begin{abstract}
Resumen
Análisis bibliométrico de la red de colaboración científica que genera la coautoría de artículos entre autores de las universidades españolas. A partir de los documentos contenidos en el Science and Social Citation Index entre 2000 y 2004, se analizaron los datos de producción más importantes aplicando el método de análisis de redes sociales, elaborando un mapa bibliométrico que permite su visualización. La red de colaboración interuniversitaria en España resultante, permite observar que está compuesta mediante la interconexión de subredes de colaboración de ámbito regional. La red de colaboración entre las universidades españolas se constituye en un gran componente con ausencia de componentes alternativos y/o nodos aislados. La colaboración entre los investigadores universitarios españoles está geográficamente localizada.
\end{abstract}

Palabras clave: Colaboración en investigación. Análisis de redes sociales. Proximidad. Estructura espacial. España. Universidades.

\section{Introducción}

La colaboración científica es uno de los mecanismos sociales claves de las prácticas contemporáneas en investigación. Los estudios bibliométricos demuestran un incremento en el número de artículos con coautoría a nivel internacional (Persson, Glänzel y Danell, 2004; Wagner y Leydesdorff, 2005), en los países de la Unión Europea (UE) (European Commission, 2003) y en España (Moya Anegón, et al., 2005).

La coautoría de artículos científicos probablemente representa la evidencia empírica que queda registrada, más importante de la interacción entre los investigadores. La coautoría es costosa en términos de esfuerzos y los autores dispuestos a colaborar de esta forma asumen sus costes implícitos. Los costes arrancan desde el mismo momento en que se tiene que responder a la pregunta inicial: ¿con quién debo colaborar?, es decir, desde el momento en que hay que seleccionar a un posible socio. Pero no

\begin{abstract}
Bibliometric analysis of scientific research co authorship network from Spanish universities. Using the documents referred in the Science and Social Citation Index from 2000 to 2004, the more important output data is analyzed applying social network analysis, and developing a bibliometric map that allows its visualization. The Spanish intrasectorial university network it's compound by the interconnection of subnetwork of regional scope. The research collaboration network between the Spanish universities constitutes a great component with absence of alternative components and/or isolated nodes. Spanish university research networks are geographically localized.
\end{abstract}

Keywords: Research collaboration. Social network analysis. Proximity. Spatial structure. Spain. Universities.

es el único coste. Para colaborar hay que compartir las ideas centrales de un proyecto, las ideas rectoras y las consecuencias que generen dichas ideas. Hay que lograr un clima de confianza, establecer una división del trabajo y compartir información y coordinar los trabajos, para finalmente redactar el escrito y distribuir y asignar el mérito en el delicado momento de las autorías de los artículos.

La coautoría a veces, también significa investigación conjunta, y se convierte en una oportunidad para el intercambio de conocimiento tácito (Heffner, 1981). La coautoría refleja la generación e intercambio de conocimiento nuevo y actual, con la que se logra una mayor autoridad desde el punto de vista epistemológico, poniendo además en evidencia la resolución de problemas conjuntos (Beaver, 2004). Sin embargo, la coautoría no abarca, ni refleja todo el rango posible de intercambios de conocimientos que se producen entre los investigadores, como son, por ejemplo, las conversaciones informales, la 
escucha de las presentaciones en congresos, o la pertenencia y asistencia a comités profesionales o reuniones de toda índole.

En España, la producción científica del sector de instituciones de educación superior ha sido analizada de forma específica en diversos artículos, tesis doctorales e informes de investigación. De entre todos ellos, hay que reseñar aquella línea de trabajos cuyo objetivo se centra, a partir exclusivamente de la producción universitaria a nivel nacional, en el análisis de aspectos concretos de la producción, como la excelencia (Moya Anegón et. al., 2004a), la citación (Camí, 2004) la interdisciplinariedad (Rovira Pato, 2006) o el rendimiento universitario, basándose en análisis con indicadores de input-output (Gómez et al., 2007).

Este trabajo selecciona un punto de vista entre los posibles. Estudia los patrones de colaboración científica mediante coautoría de artículos en un macro nivel, España, desde un punto de vista interno; esto es, la colaboración que se produce entre las universidades españolas. La coautoría, en consecuencia es la principal variable dependiente.

En particular, nos interesa responder a preguntas como:

1. ¿Qué grado de cooperación existe entre los investigadores universitarios de las diferentes Comunidades Autónomas (CCAA)?

2. ¿Qué tipos de colaboración prevalece?

3. ¿Qué tendencias pueden desprenderse?

4. ¿Qué universidades colaboran más y qué tipo de colaboración predomina?

5. La proximidad geográfica, ¿juega algún papel en la configuración de la red interuniversitaria? ¿existen subredes regionales?

Mediante la aplicación de algunas técnicas de análisis de redes, trataremos de caracterizar los nodos principales y visualizar la red y disposición de los nodos resultante.

\section{Material y métodos}

La fuente original de los datos para elaborar los indicadores bibliométricos ha sido el ISI Web of Science (WOS, 2006), un producto de la empresa Thomson Scientific en el que están incluidas las bases de datos objeto de explotación: 1) Science Citation Index Expanded (SCl- Expanded), especializada en Ciencias y Medicina; 2) Social Science Citation Index (SSCl), especializada en Ciencias Sociales; y 3) Arts and Humanities Citation Index $(\mathrm{AHCl})$.

\subsection{Datos}

De las bases mencionadas se han extraído los registros correspondientes a los años 2000 al 2004, ambos inclusive, de aquellos documentos en los que apareciera Spain en el campo Address. La extracción de la información para la realización del estudio se ha hecho a partir de la base de datos completa en la versión en línea del WOS. Siguiendo los criterios expuestos, los resultados iniciales de las búsquedas arrojaron un total de 151.600 documentos publicados en universidades españolas. Posteriormente se realizó un proceso de normalización del campo dirección, para determinar con fiabilidad a qué institución y por extensión, a qué Comunidad Autónoma pertenecía cada uno de los registros bibliográficos. Tras ese proceso, los correspondientes al sector universitario quedaron recogidos en un subconjunto de 100.710 documentos de todo tipo artículos, resúmenes de congresos, revisiones, cartas, editoriales y críticas de libros.

Con estos datos, se ha construido una base relacional en la que las tablas principales han sido las de documentos, citas, instituciones, palabras clave, categoría, clase, que a su vez han sido organizadas en campos y se han establecido las correspondientes relaciones entre las mismas.

\subsection{Normalización de los datos}

Para poder analizar los datos, ha sido preciso llevar a cabo una normalización de ciertos campos de las bases de datos, especialmente el de los nombres de las instituciones de los autores firmantes de los trabajos. El campo Address suele constar de información en cuatro niveles: la organización principal, un departamento de la organización, la ciudad y el país. En muchos casos, sólo hay tres niveles, excluyendo el nivel departamental o el institucional. El país suele estar bien normalizado y la ciudad puede normalizarse a partir de los códigos postales. En todos estos niveles podemos encontrar un gran número de variantes. Este ha sido uno de los problemas que se ha tenido que solucionar ya que afectaba directamente a la creación de las relaciones entre las ubicaciones de las diferentes instituciones y organismos productoras productores de información científica de las Comunidades autónomas. Para poder establecer la correspondencia entre las sedes de las organizaciones y la universidad correspondiente se han localizado las variantes en la forma de cada una de las instituciones productoras, se ha adoptado y se han adscrito al centro y a la Comunidad correspondiente mediante procedimientos semiautomáticos, una vez creado un 
fichero de autoridades, en el que las variaciones aceptables de una dirección remiten al adoptado como entrada aceptada.

\subsection{Tratamiento de los datos}

Para la clasificación de los datos bibliográficos se han considerado las siguientes variables: temporal, temática, geográfica, sectorial e institucional. El análisis se centra en un nivel nacional, como se corresponde con la producción de las 70 universidades españolas, públicas y privadas, inscritas en el Registro Nacional de Universidades, Centros y Enseñanzas del Ministerio de Educación y Ciencia en junio de 2007.

\subsection{Distribución temporal}

Para asignar un documento a un año, se ha tomado como referencia el año de publicación del número de la revista en la que aparece dicho documento. Esta información es propia de la referencia bibliográfica y permite temporalizar los análisis bibliométricos. El objetivo inmediato ha sido agrupar los datos por años para ver la evolución anual de cualquiera de los indicadores bibliométricos utilizados para el estudio.

\subsection{Distribución geográfica}

Para la distribución geográfica se ha seguido la división administrativa española por Comunidades autónomas, lo que permite la realización de comparaciones entre las distintas regiones, y de éstas con España. La producción de las ciudades autónomas de Ceuta y Melilla ha sido incluida en la producción andaluza.

\subsection{Modos de recuento, indicadores y representación gráfica}

El conjunto de indicadores para conocer los aspectos cuantitativos de los resultados científicos de la producción con colaboración universitaria de España está basado en el recuento de publicaciones. El Indicador Ndoc indica el número de documentos de cualquier tipo, en los que intervenga un autor que pertenezca a una institución universitaria española, recogidos en las bases de datos del ISI Web of Science.

Las bases de datos de Thomson contienen datos de las afiliaciones institucionales de los investigadores que reciben el reconocimiento como autores en los artículos indizados en las bases de datos. Sin embargo, salvo los nombres y las direcciones institucionales de los autores, los registros no contienen ninguna información adicional sobre las disciplinas de las que tratan sus contribuciones, nacionalidad, edad, sexo, rango administrativo o status dentro de sus organizaciones. Aunque en el trabajo se utiliza el término "coautoría", por este término debe entenderse autoría institucional, es decir, la afiliación institucional de los autores (Melin y Persson, 1996). El método, no es perfecto, ya que al hacerse este tipo de atribución, basándose en las direcciones institucionales de los autores, si se omiten en la revista original la dirección de un colaborador, no se podrá atribuir la coautoría ni a la institución, ni al país en cuestión, en el caso que el artículo sea el resultado de la colaboración internacional.

Los indicadores se han desagregado por tipos de colaboración en las siguientes categorías: sin colaboración, trabajos con un único autor individual; intrasectorial, trabajos firmados por más de una universidad; nacional, trabajos firmados por más de una institución nacional cualquiera que sea su sector de actividad; e internacional, trabajos firmados por autores de algún país distinto de España. La nacional puede ser a su vez, subdividida en interregional, producida en colaboración con instituciones que no pertenecen a la Comunidad Autónoma, e intrarregional, producida en colaboración con instituciones de la misma Comunidad Autónoma.

Cuando un artículo es el producto de una colaboración entre autores de diferentes instituciones, la atribución del trabajo se puede asignar básicamente de dos formas diferentes. Como en este estudio se usa el nivel de la dirección institucional, las posibilidades de medición de la intensidad se reducen a dos: a) atribuir todo el mérito a cada uno de los firmantes: contabilizando "1" a cada autor firmante o institución (método de contabilidad completo); o b) dividir la unidad por el número de autores, países, instituciones, que participan en la autoría del trabajo en colaboración, asignando una fracción del mérito a cada uno de ellos (método de contabilidad fraccionado).

Cuando se opta por esta forma de asignación (contabilidad completa), como en este caso, la atribución de un único documento puede ser doble o triple según el tipo de agregación que se compute. Este tipo de recuento tiene el inconveniente de la duplicación de los documentos, que hacen que los sumatorios sean superiores al total real de los documentos. Aunque esas diferencias en los agregados respecto del número total de documentos "reales" (Ndoc), no son importantes, para aclarar esa circunstancia en algunas tablas se presentan los datos con dos filas referidas a los totales: total sin solapamiento (total SS) y el total con solapamiento (total CS). 
Las redes de coautoría deben ser estudiadas con múltiples medidas, independientemente de si la red describe la coautoría internacional, interinstitucional, interregional, interdepartamental o personal. Sólo así puede comprenderse la estructura de colaboración subyacente.

De las múltiples medidas de la coautoría, uno de los aspectos a considerar es la fuerza que existe en la relación o vínculo que establecen indirectamente las universidades que colaboran entre sí y el grado de atracción entre las distintas universidades coautoras de trabajos científicos. La existencia de una colaboración entre dos naciones, instituciones o personas, implica que existe reciprocidad -si la universidad $X$ colabora con universidad $Y$, la segunda colabora con la primera-, pero no indica el grado de dependencia de una sobre otra. El grado de dependencia puede variar entre las distintas instituciones, ya que los grados de colaboración puede que no sean simétricos.

La confirmación o reciprocidad es una propiedad importante de los vínculos en el análisis de redes. La medida de la confirmación no está definida por la existencia del enlace, sino por el grado en el que los diferentes nodos poseen o no el mismo valor de reciprocidad (Tichy y Tushman, 1979).

Para representar esta desemejanza en el grado de colaboración entre universidades, se ha calculado la tasa de colaboración asimétrica, y se ha representado gráficamente en el mapa de colaboración interuniversitario construido indicándose la asimetría mediante el sentido diferente de las puntas de las flechas que vinculan los nodos. La idea de este indicador procede del Índice de afinidad empleado para los cálculos de relaciones asimétricas entre dos países (Zitt, 2000), pero adaptándolo para el cálculo de la colaboración asimétrica entre universidades.

Se ha calculado del modo siguiente, con dos fórmulas que miden el sentido de la colaboración entre dos nodos cualesquiera:

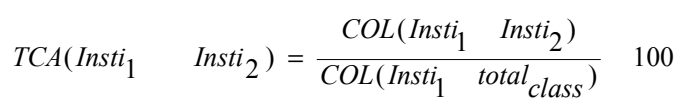

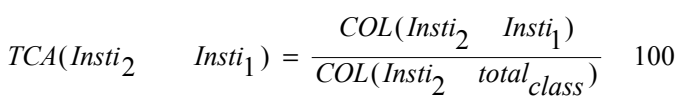

Debido a la naturaleza compleja de los datos que proceden de estudios métricos, éstos deben ser transformados en representaciones gráficas. Esta circunstancia es inducida por el tipo de datos que se obtiene en muchos estudios en los que se seleccionan unidades de análisis, como la coautoría, que expresan por sí mismas relaciones entre los documentos contenidos en las bases de datos analizadas. Por eso, además de las tablas estadísticas y gráficos que se usan en el presente trabajo, se ha añadido un mapa obtenido mediante el empleo de técnicas de visualización derivadas del análisis de redes sociales (Freeman 2000).

Para representar el mapa de colaboración interuniversitario a partir de los datos obtenidos, se ha recurrido al algoritmo de representación gráfica Kamada-Kawai (Kamada, 1989), incluido en el programa de análisis de redes Pajek (Batagelj y Mrvar, 1997).

Este algoritmo diseña la red de nodos asumiendo que los enlaces entre ellos se comportan físicamente como muelles, existiendo una longitud de muelle ideal, que se corresponde con la distancia entre los nodos y una fuerza en el muelle. Los nodos se pueden ubicar en un espacio bi o tridimensional y el conjunto del sistema, se hace evolucionar, con el fin de disminuir la energía de los muelles. Así, siguiendo a Vargas-Quesada y Moya Anegón (2007, p. 51):

Algorítmicamente, para evitar problemas de computación, se calcula la evolución de cada nodo por separado, en lugar de todo el conjunto. Es decir, se fijan todos los nodos salvo aquel que más energía acumula y se deja evolucionar hasta que la energía acumulada sea menor que un determinado límite y lo volvemos a fijar. A continuación, se vuelve a escoger el nodo que acumula más energía, se le hace evolucionar y se vuelve a fijar. Y así sucesivamente hasta que ninguno de los nodos que componen la red, acumule una energía superior al límite.

Después de haberse empleado el algoritmo, la distancia de los enlaces se visualiza fácilmente porque correlaciona muy bien con la distancia física.

La generación de la red a partir de los datos brutos de coautoría (Leydesdorff y Vaughan, 2006), permite posteriormente enriquecerla a partir de anotaciones de los nodos (nombre), tamaños (indicando por ejemplo, valores de producción), colores (valores de sectores, comunidades autónomas o países), etc. Este tipo de grafos pueden incluir más de tres dimensiones, multiplicándose el número de variables que pueden añadirse y dando origen a representaciones hípervariadas.

\section{Resultados}

Desde el año 1978, España se organiza territorialmente en municipios, en provincias y en diecisiete Comunidades autónomas y con dos territorios, Ceuta y Melilla que son considerados ciudades autónomas. Esta configuración ha modificado radicalmente el sistema de gobierno 
en las políticas de ciencia y tecnología (Cruz Castro, Sanz Menéndez y Romero, 2004), pasando las CCAA a tener un papel decisivo en el control, financiación y orientación de las actividades de investigación, ya que desde mediados de los años noventa del pasado siglo, las universidades públicas han ido a pasando al control y tutela de las CCAA; y su financiación, a ser controlada por éstas. En la Tabla I se muestran los datos de la producción universitaria por tipos de colaboración y Comunidad Autónoma. A nivel nacional, seis de cada diez artículos se escriben en colaboración, y tres de cada diez cuentan con colaboradores internacionales. La colaboración nacional es la más frecuente, por encima de la que se realiza exclusivamente con profesores que trabajan en universidades, que es la menos frecuente de entre todos los tipos analizados.

Tipos de colaboración (\%)

\begin{tabular}{lrrrrrr} 
CCAA & & & & & & \\
& & & & & & \\
\cline { 5 - 6 } & & En col. $(\%)$ & Sin col. & Intrasectorial & Nacional & Internacional \\
\hline Andalucía & 148840 & $8534(57.51)$ & $6306(42.49)$ & $2285(15.40)$ & $3904(26.31)$ & $4630(31.20)$ \\
Aragón & 3528 & $2376(67.35)$ & $1152(32.65)$ & $408(11.56)$ & $1180(33,45)$ & $1196(33.90)$ \\
Asturias & 3253 & $2006(61.67)$ & $1247(38.33)$ & $406(12.48)$ & $1078(33.14)$ & $928(28.53)$ \\
Baleares & 1034 & $668(64.60)$ & $366(35.40)$ & $139(13.44)$ & $246(23.79)$ & $422(40.81)$ \\
Canarias & 2757 & $1732(62.82)$ & $1025(37.18)$ & $410(14.87)$ & $835(30.29)$ & $897(32.54)$ \\
Cantabria & 1466 & $1052(71,76)$ & $414(28,24)$ & $222(15.14)$ & $539(36.77)$ & $513(34,99)$ \\
Castilla la Mancha & 1444 & $841(58.24)$ & $603(41.76)$ & $345(23,89)$ & $480(33.24)$ & $361(25.00)$ \\
Castilla León & 5161 & $3161(61,25)$ & $2000(38.75)$ & $864(16.74)$ & $1569(30.40)$ & $1592(30.85)$ \\
Cataluña & 23178 & $16767(72,34)$ & $6411(27,66)$ & $2243(9.68)$ & $7958(34.33)$ & $8809(38.01)$ \\
Extremadura & 1651 & $857(51.91)$ & $794(48.09)$ & $286(17.32)$ & $378(22.90)$ & $479(29.01)$ \\
Galicia & 7880 & $4545(57.68)$ & $3335(42.32)$ & $1294(16,42)$ & $2120(26.90)$ & $2425(30.77)$ \\
Madrid & 20356 & $13602(66.82)$ & $6754(33.18)$ & $2897(14.23)$ & $7023(34.50)$ & $6579(32.32)$ \\
Murcia & 2855 & $1624(56.88)$ & $1231(43.12)$ & $571(20.00)$ & $889(31.14)$ & $735(25.74)$ \\
Navarra & 2810 & $1889(67.22)$ & $921(32.78)$ & $364(12.95)$ & $1030(36.65)$ & $859(30.57)$ \\
País Vasco & 4086 & $2434(59.57)$ & $1652(40.43)$ & $661(13.73)$ & $1110(27.17)$ & $1324(32.40)$ \\
Rioja & 326 & $203(62.27)$ & $123(37.73)$ & $89(27.30)$ & $138(42.33)$ & $65(19.94)$ \\
Valencia & 12091 & $7847(64.90)$ & $4244(35.10)$ & $1867(15.44)$ & $3868(31.99)$ & $3979(32.91)$ \\
Total CS & 108716 & $70138(64.51)$ & $38578(35.49)$ & $15251(14.03$ & $34345(31.59)$ & $35793(32.92)$ \\
Total SS & 100710 & $62479(62,02)$ & $38231(37.96)$ & $9814(9.74)$ & $28802(28.60)$ & $33677(33.44)$ \\
\hline
\end{tabular}

Tabla I. Producción universitaria por tipos de colaboración y CCAA. Tanto por ciento entre paréntesis

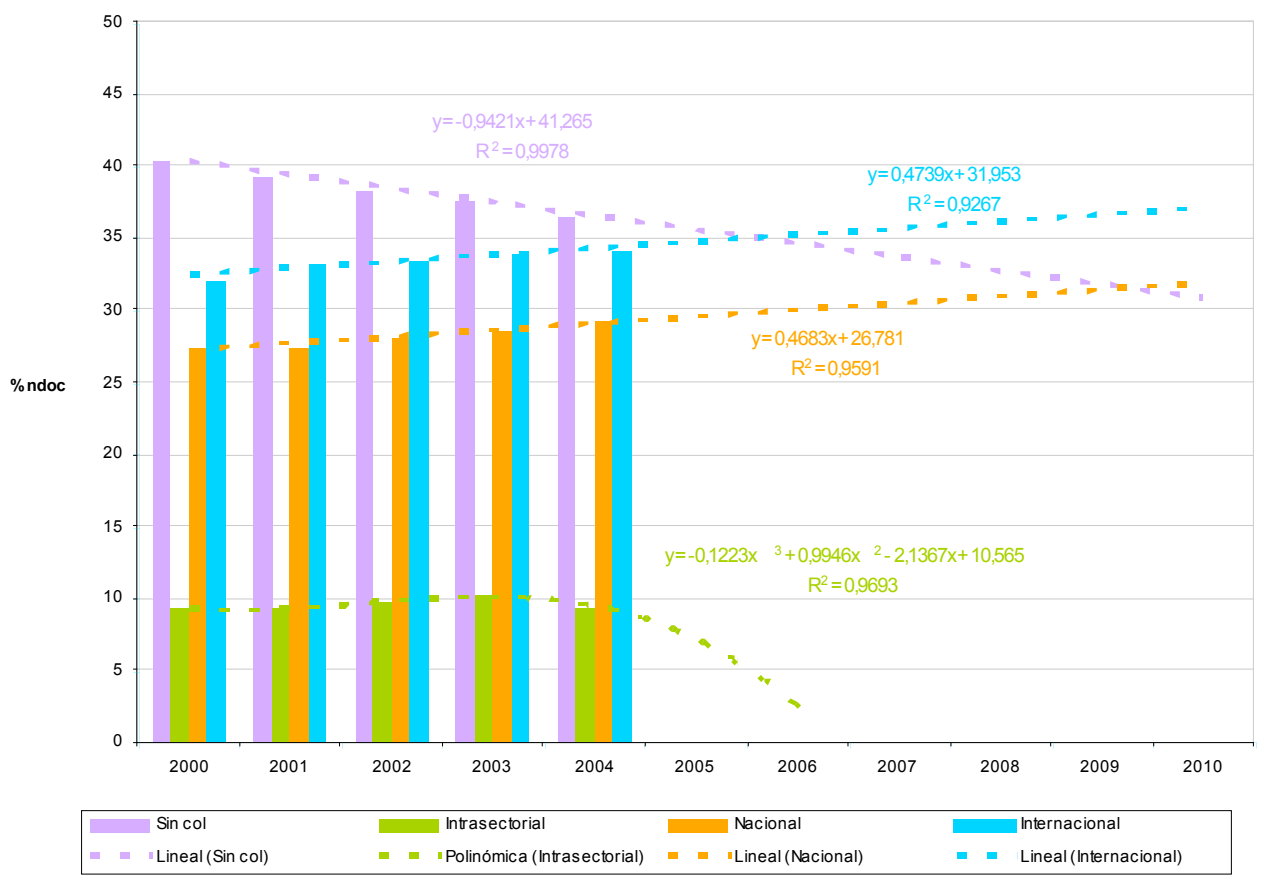

Figura 1. Producción científica universitaria española por tipos de colaboración y tendencias (2000-2010) 
Los resultados de las universidades agrupadas por Comunidades Autónomas indican que los investigadores más activos por su orientación hacia la colaboración, proceden de Aragón, la Comunidad Balear, Cantabria, Cataluña, Madrid, Navarra y Valencia. Por debajo de la media nacional (64\%) se encuentran aquellos otros que trabajan en universidades de radicadas en Andalucía, Asturias, Canarias, Castilla La Mancha, Castilla León, Extremadura, Murcia, País Vasco y Rioja. Son también entre las últimas Comunidades mencionadas, en donde se encuentran con más frecuencia los profesores e investigadores universitarios que trabajan en solitario, sin buscar colaboradores para sus investigaciones: Extremadura (48\%), Murcia (43\%), Andalucía (42\%), Galicia $(42 \%)$ y País Vasco $(40 \%)$.

Por último, es de destacar el activo comportamiento de los investigadores de las Baleares $(40 \%)$, Cataluña (38\%), por el logro de numerosos vínculos establecidos con investigadores extranjeros, que otorga a estas Comunidades, los mayores porcentajes de trabajos firmados con colaboradores internacionales en el quinquenio estudiado. En el otro extremo se hayan quienes no logran alcanzar la media nacional: aquellos que trabajan en las siguientes Comunidades: La Rioja (19\%), Castilla la Mancha (25\%), Murcia (25\%), Castilla y León (30\%), Asturias y Extremadura.

Las tendencias de los distintos tipos de colaboración universitaria que se pueden esperar en España, ("Figura 1", en la página anterior), muestran que, de mantenerse la tendencia, la colaboración internacional y la nacional, son las que tenderán a incrementarse en los próximos años. Los trabajos sin colaboración a nivel nacional respecto de la producción total, son los que claramente tienden a decrecer $\left(R^{2}=0.997\right)$.

También tiene interés observar con mayor detalle en este retrato macroscópico, las diferencias en la colaboración nacional entre instituciones existentes en la propia Comunidad Autónoma y la ajenas a la misma. En la Figura 2 se representa la producción nacional con colaboración, desagregada por instituciones de la misma Comunidad Autónoma (intrarregional) y la producida con instituciones radicadas en otras Comunidades (interregional).

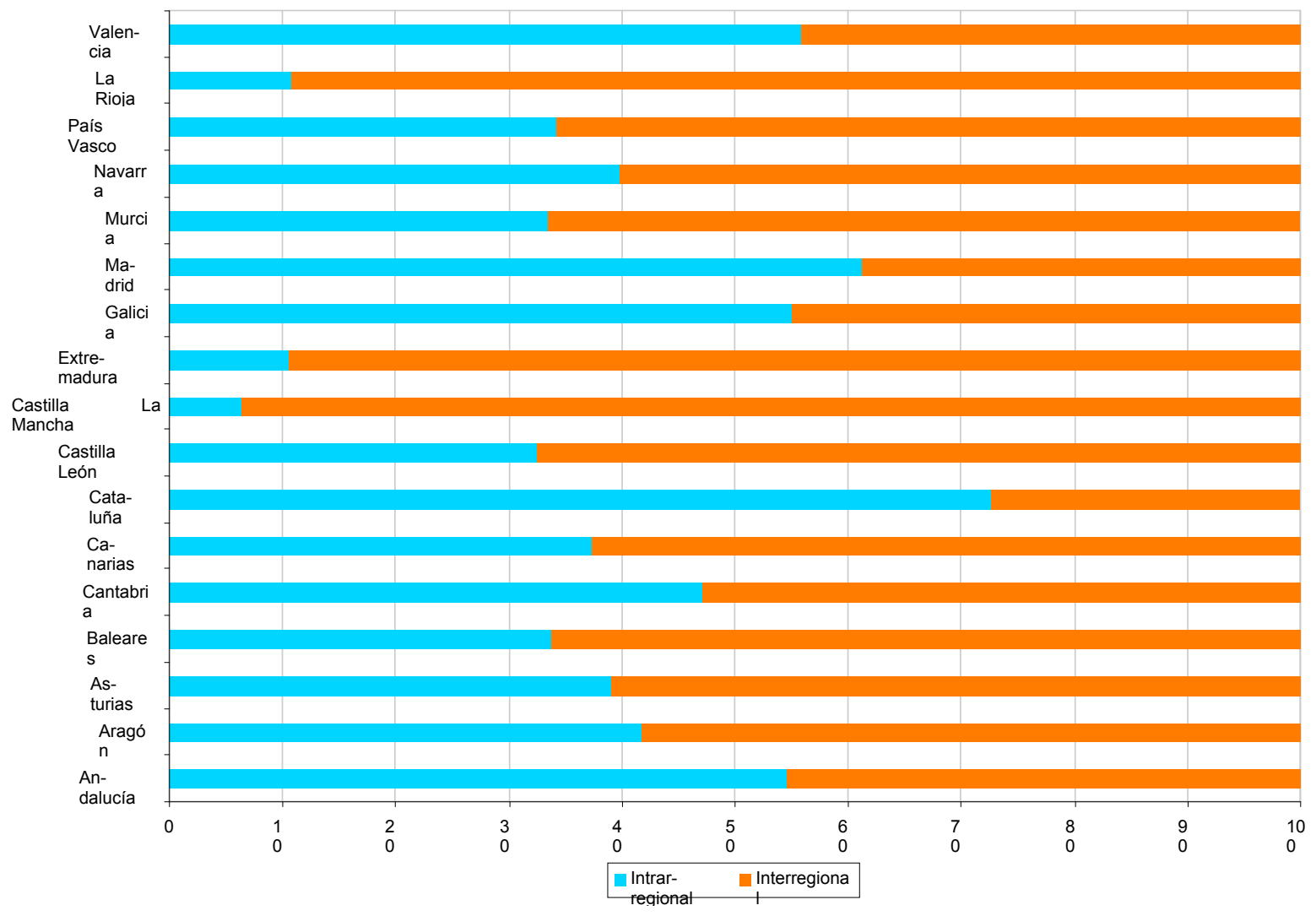

Figura 2. Colaboración intraregional e interregional por CCAA en tantos por ciento 


\begin{tabular}{lrrrrrr} 
& & & \multicolumn{3}{c}{ Tipos de colaboración (\%) } \\
\cline { 5 - 7 } Universidad & Ndoc & En col (\%) & Sin col & Intrasectorial & Nacional & Internacional \\
\hline U Barcelona & 10596 & $8130(76.73)$ & $2466(23.27)$ & $986(9.31)$ & $4213(39.76)$ & $3917(36.97)$ \\
U Complutense & 8690 & $5707(65.67)$ & $2983(34.33)$ & $1397(16.08)$ & $3023(34.79)$ & $2684(30.89)$ \\
UA Barcelona & 6195 & $4800(77.48)$ & $1395(22.52)$ & $819(13.22)$ & $2583(41.69)$ & $2217(35.79)$ \\
U Valencia & 6020 & $4241(70.45)$ & $1779(29,55)$ & $1061(17.62)$ & $2122(35.25)$ & $2119(35.20)$ \\
UA Madrid & 5341 & $4088(76.54)$ & $1253(23.46)$ & $589(11.03)$ & $2087(39.08)$ & $2001(37.46)$ \\
U Santiago & 4926 & $3015(61.21)$ & $1911(38.79)$ & $842(17.09)$ & $1365(27.71)$ & $1650(33.50)$ \\
U Granada & 4557 & $2761(60.59)$ & $1796(39.41)$ & $962(21.11)$ & $1345(29.52)$ & $1416(31.07)$ \\
U P Vasco & 4028 & $2397(59.51)$ & $1631(40.49)$ & $542(13.46)$ & $1084(26.91)$ & $1313(32.60)$ \\
U Sevilla & 3715 & $2202(59.27)$ & $1513(40.73)$ & $570(15.34)$ & $1028(27.67)$ & $1174(31.60)$ \\
U Pol Barcelona & 3601 & $2262(62.82)$ & $1339(37.18)$ & $679(18.86)$ & $864(23.99)$ & $1398(38.82)$ \\
U Zaragoza & 3528 & $2376(67.35)$ & $1152(32.65)$ & $408(11.56)$ & $1180(33,45)$ & $1196(33,90)$ \\
U Oviedo & 3253 & $2006(61.67)$ & $1247(38.33)$ & $406(12.48)$ & $1078(33.14)$ & $928(28.53)$ \\
U Pol Valencia & 2725 & $1711(62.79)$ & $1014(37.21)$ & $590(21.65)$ & $935(34.31)$ & $776(28.48)$ \\
U Pol Madrid & 2680 & $1753(65.41)$ & $927(34.59)$ & $759(27.99)$ & $990(36.94)$ & $763(28.47)$ \\
U Murcia & 2483 & $1378(55.50)$ & $1105(44.50)$ & $455(18.32)$ & $740(29.80)$ & $638(25.69)$ \\
U Vigo & 2390 & $1460(61.09)$ & $930(38.91$ & $649(27.15)$ & $854(35.73)$ & $606(25.36)$ \\
U Málaga & 2060 & $1108(53.79)$ & $952(46.21)$ & $308(14.95)$ & $539(26.17)$ & $569(27.62)$ \\
\hline
\end{tabular}

Tabla II. Número de documentos publicados por universidades españolas con y sin colaboración (ndoc $>2000)$

Estas colaboraciones se pueden dar con instituciones de otros sectores diferentes del universitario, como son las empresas, los hospitales, las entidades públicas de investigación, unidades de la Administración y los organismos pertenecientes al Consejo Superior de Investigaciones Científicas.

En las fluctuaciones de porcentajes entre un tipo y otro en cada Comunidad Autónoma influyen, obviamente, factores como la existencia de más de una universidad, el grado de diversidad de instituciones ubicadas en cada una de las Comunidades, consecuencia, a su vez, de la construcción institucional de la política científica y tecnológica española (Sanz Menéndez, 1997), las condiciones socio-económicas regionales desiguales, el diferente nivel de desarrollo relativo y el peso de los diversos actores de la I+D en la Comunidad respectiva.

Las cuatro comunidades universitarias con mayor hábito de producción con colaboradores de su propia CCAA son las mayores productoras a nivel nacional, pero con porcentajes diferentes. Los investigadores radicados en Cataluña, firman en $70 \%$ de su producción nacional, con instituciones radicadas en su propia Comunidad, seguidas de Madrid (61\%), Valencia (55\%) y Andalucía (54\%).

Frente a estas Comunidades donde predominan los lazos de carácter endógeno, existen otras en las que predominan con claridad los vínculos de colaboración con instituciones establecidas fuera de su ámbito territorial. Son los casos de Castilla la Mancha (93\% de su producción), la
Rioja (89\%). En posiciones intermedias se sitúan Cantabria, Asturias, Navarra y Aragón.

Tan importante como los resultados por Comunidades Autónomas, son los datos individuales de las universidades españolas, ya que ayudan a hacer más visibles las diferencias entre los centros más prolíficos y los que lo son menos. A tal fin se presentan los datos de las universidades más productivas y con mayores porcentajes de producción elaborada en colaboración, así como los porcentajes resultado de su actividad de colaboración con otros centros nacionales e internacionales. Se presentan aquellos centros con una producción superior a 2.000 documentos en el período, que suman el $70.6 \%$ de la producción nacional total (tabla II, en la página anterior).

Las más destacadas por su producción con colaboración, son la Universidad Autónoma de Barcelona $(77 \%)$, la Universidad Central de Barcelona $(76 \%)$, la Universidad Autónoma de Madrid (76\%), y la Universidad de Valencia $(70 \%)$. Estos porcentajes se han calculado sobre los 30822 documentos publicados en el período por estas universidades $(28.3 \%$ del total nacional).

Si atendemos a la colaboración internacional, la Universidad Politécnica de Barcelona, la Universidad Pública del País Vasco, la de Santiago de Compostela, y tres universidades andaluzas, Granada, Sevilla y Málaga, son las que tienen los mejores porcentajes de trabajos con colaboración internacional, hasta al punto que superan a los que publican con colaboración nacional; pero también tres de ellas, Málaga (46\%), Sevi- 
Ila (40\%), la pública del País Vasco $(40 \%)$, junto con la de Murcia (44\%), son las que producen más trabajos en solitario.

La colaboración nacional es predominante en las cuatro universidades que poseen el mayor porcentaje de producción en colaboración mencionadas más arriba. Las que obtienen el mejor diferencial, comparando la producción con colaboración nacional e internacional son las de Valencia y Autónoma de Madrid -0.05 y -1.62 , respectivamente.

La tabla III, muestra los resultados del análisis de redes sociales efectuado sobre la red de colaboración intrasectorial durante los 5 años que comprende este estudio. El análisis de redes sociales es utilizado como metodología para el análisis de las redes de coautoría (Newman, 2001) y nos proporciona un número elevado de datos estadísticos (Otte y Rousseau, 2002).

\begin{tabular}{lc}
\hline Datos estadísticos de la red & Valores de la red \\
\hline Número de nodos & 69 \\
Número de vínculos & 2204 \\
Tamaño del componente K-core & 40 \\
Densidad de la red & 0.46 \\
Grado medio & 63.8 \\
Diámetro & 3 \\
Grado de centralización & 0.46 \\
Grado de intermediación & 0.06 \\
Grado de cercanía & 0.66 \\
Coeficiente de agrupamiento & 0.34 \\
\hline
\end{tabular}

Tabla III. Análisis de redes sociales de las universidades españolas

El número de nodos representa el número de universidades. El tamaño del $K$-core, o de la parte más densa de la red, alcanza a 40 nodos. La densidad, que es una medida de la cohesión, tiene un valor de 0.46 , lo que indica que el $46 \%$ de todos los posibles vínculos o enlaces entre las universidades están presentes, lo que sugiere una elevada densidad. El grado medio es otra medida estructural de cohesión de la red y mide la media de grado, o el número medio de vínculos con los que las universidades se vinculan entre sí. La distancia geodésica entre los extremos de la red, es de tres, lo que confirma, de nuevo, el grado de densidad de la red.

Tanto los valores de centralidad de grado, como los de cercanía, indican que estamos ante una red fuertemente interconectada. La centralidad de cercanía de los nodos se mueve en un rango entre 0.42 y 0.87 y 51 nodos de los $69(74 \%$ del total de nodos), entre 0.57 y 0.87 . Sin embargo, el bajo valor de centralidad de intermediación de la red, indica que hay varios nodos invisibles, en concreto son 10 , porque tienen 0 valor de intermediación; es decir, no median entre otros vértices $y$, por lo tanto, se constituyen en la periferia de la red.

El último valor de la tabla se refiere al coeficiente de agrupamiento, que no es muy alto (0.34). Los agrupamientos son grupos existentes en las redes en los que se pueden producir vínculos redundantes. El coeficiente mide la probabilidad de que un nodo pertenezca a un agrupamiento. Este valor indica hasta qué punto son densas las relaciones entre el círculo de colaboradores de un nodo determinado. A nivel global, la red de universidades constituye un único componente; y eso significa que todos los nodos tienen vínculos entre sí y están interconectados como un todo, sin existir ni vértices, ni subredes aisladas. El agrupamiento se produce cuando existe en alguna parte de la red múltiples vinculaciones que determinan una zona de intensa cohesión, por ejemplo, en nuestro caso, por la posible existencia de políticas públicas que incentivaran de algún modo, algún tipo de colaboración.

El mapa de colaboración intrasectorial de las universidades españolas se ha representado en la figura 3, en la página siguiente. En la representación, el tamaño de los nodos es proporcional al volumen de producción con colaboración intrasectorial y cada nodo que representa una universidad, tiene asignado un color que la identifica con la Comunidad Autónoma a la que pertenece. El tipo de red construida resultante de la colaboración intrasectorial entre las universidades de España permite observar que está compuesta mediante la interconexión de subredes de colaboración de ámbito regional.

Existe un núcleo de gran tamaño compuesto por la subred de la Comunidad de Madrid, con tres nodos con el mayor grado, la Universidad Complutense, Autónoma de Madrid y Politécnica, siendo las dos primeras las que ocupan la mayor centralidad, en función del grado. Alrededor de la universidad Complutense existe una pequeña constelación de universidades satélites compuesta por pequeñas universidades privadas (Francisco de Vitoria, San Pablo CEU, Europea, Pontificia de Comillas); públicas de reciente creación, como la universidad Rey Juan Carlos o universidades singulares como la Universidad Nacional de Educación a Distancia. El sentido de los vínculos demuestra su dependencia, en términos de colaboración, de los investigadores que trabajan en la Complutense, que se convierte en su socio preferente en todos los casos. 


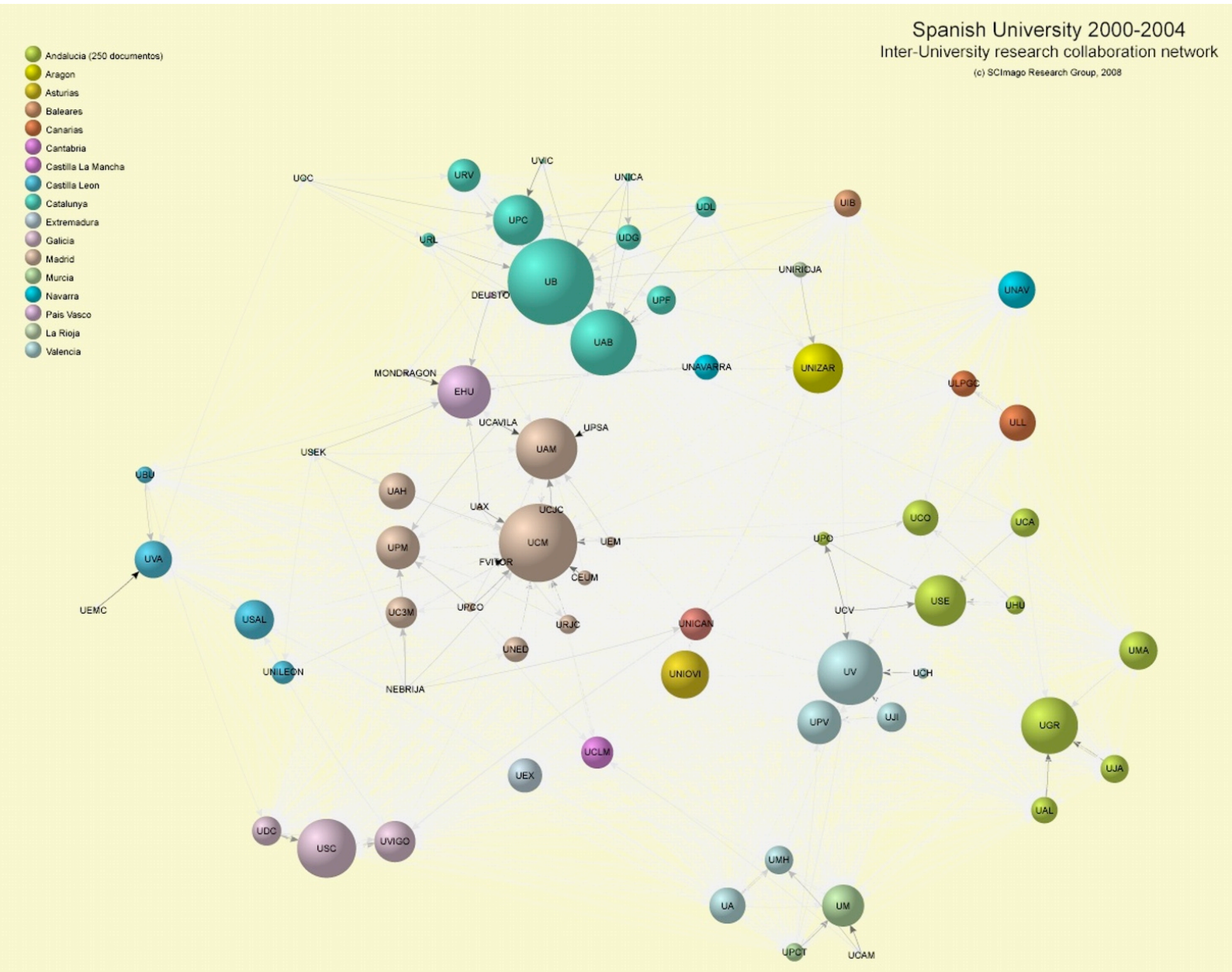

Figura 3. Red de colaboración asimétrica de las universidades españolas (2000-2004)

Algo similar sucede en la configuración de la subred catalana. En torno a las universidades más antiguas, Central, Autónoma y Politécnica, se hallan las universidades creadas a partir de los inicios de la década de los años noventa del pasado siglo: universidades de Lérida, Gerona, Ramón Llull, Internacional, Rovira Virgili o Pompeu Fabra. Al igual que sus homólogas madrileñas, su producción es coautorada fundamentalmente con profesores e investigadores de las universidades más veteranas.

Próximas a la sub red catalana, aparecen las universidades Pública de Navarra, Zaragoza, Rioja, e Islas Baleares. Mientras, la universidad privada de Navarra se sitúa en una posición periférica y sin constituir un agrupamiento específico.

La subred de la Comunidad Valenciana, presenta una singularidad, que no es una excepción. Mientras que las ubicadas en la provincia de Valencia y Castellón, Politécnica, Valencia, Jaume I, Cardenal Herrera CEU, Católica de
San Vicente Mártir, mantienen vínculos de colaboración estrechos, los profesores e investigadores de la universidad de Alicante, que administrativamente pertenecen a la mencionada Comunidad, sin embargo colaboran más intensamente con universidades más próximas por localización, como son las que pertenecen a la región de Murcia: Católica de San Antonio, Murcia y Politécnica de Cartagena.

La subred andaluza tiene dos núcleos diferentes. El primero, el encabezado por la Universidad de Sevilla, alrededor de la cual se sitúan las universidades de Córdoba, Cádiz, Huelva y Pablo de Olavide; y en el extremo inferior derecha del mapa, se sitúa el otro núcleo integrado por la Universidad de Granada, con las Universidades de Málaga, Jaén y Almería más próxima a la primera.

La proximidad geográfica también parece ser una característica en la configuración de la organización espacial de las universidades gallegas. En este caso es la Universidad de Santiago 
de Compostela la que actúa como núcleo con la que se vinculan Coruña y Vigo.

En el caso de Castilla y León, que cuenta con siete universidades, se han conformado dos subconjuntos: el que integran las universidades de Salamanca y León, y el que componen la Universidad de Valladolid, Burgos y Europea Miguel de Cervantes. La excepción en esta Comunidad recae en la universidad Católica de Ávila, integrada en la "órbita" de las universidades madrileñas, en concreto la universidad Autónoma de Madrid.

Por último, hay que concluir con esta descripción mencionando los casos de las universidades de Cantabria y de Oviedo, con vínculos estrechos de colaboración y que aparecen en la parte central del mapa, cerca también de las universidades de las Palmas de Gran Canarias y La Laguna y de Extremadura y Castilla la Mancha.

\section{Discusión}

Las prácticas de colaboración universitaria, reflejada desde la perspectiva institucional, muestran una tendencia a incrementarse en términos nacionales e internacionales y a disminuir en los trabajos sin colaboración, de acuerdo con las tendencias internacionales.

Las universidades públicas tienen mejores resultados de colaboración que las universidades privadas; y las más antiguas por su fecha de creación, mejores que las más recientes. Los investigadores que trabajan en universidades de Cataluña, en particular los de Barcelona son los más activos a la hora de colaborar con otros colegas. En general, las universidades más antiguas tiene las composiciones más heterogéneas de departamentos, abarcan mayor número de especialidades y cuentan con mayor número de doctores como profesores permanentes.

Las universidades ubicadas en Comunidades con una orientación o predominio de su actividad económica localizada en el sector primario, como Andalucía, Castilla la Mancha, Extremadura, Rioja o Murcia, tienden a colaborar con colegas de otras Comunidades autónomas, más que con los propios compañeros de otras universidades ubicadas en la misma Comunidad, en el caso que las haya (en todas, salvo en La Rioja).

La configuración de una red, integrada por un solo componente, subdivididos en varios subgrupos, con un conjunto de universidades que actúan como periferias, pertenecientes a esos subgrupos y un tercer grupo que lo integrarían el resto de universidades, parece acomodarse, al menos desde el punto de vista de la representación, al modelo de subgrupos coherentes, descritos de este modo en la literatura sobre redes sociales (Everett y Borgatti, 1999).

Los resultados sugieren además, que la proximidad geográfica juega un importante factor en la configuración espacial de las redes entre los investigadores de las universidades españolas cuando colaboran entre sí.

Cuando se ha investigado mediante trabajos bibliométricos el papel que juega la proximidad geográfica en la producción del conocimiento, se ha detectado que la proximidad geográfica tiene un efecto positivo en la intensidad y frecuencia de la colaboración científica.

Así Sylvan Katz, al analizar la colaboración entre universidades de Canadá, Australia y Reino Unido, generó la expresión matemática de la relación entre las distancias que separan dos universidades y el número de sus colaboraciones, observando que éstas decrecen a medida que aumenta la distancia (Katz, 1994). En colaboración con Smith (Smith y Katz, 2002), concluyen que "la colaboración institucional entre instituciones de educación superior en el Reino Unido, se produce en un radio de 60 y $80 \mathrm{Km}$. y entre 80 y $100 \mathrm{Km}$. cuando son fuera del gran Londres".

Liang y Zhu (2002) han analizado la coautoría interregional de artículos científicos a partir de la producción científica nacional de China y concluyen que la proximidad geográfica es uno de los factores más importantes que afectan a la investigación interregional.

Más recientemente, Okubo y Zitt (2004) analizan los patrones de coautoría entre algunas regiones francesas con otras adyacentes y pertenecientes a otros países de la Unión Europea; y una de sus conclusiones es que los "autores de otras regiones vecinas [extranjeras] tienen más oportunidades de ser elegidos [por autores franceses] como socios preferentes que los europeos de otras regiones de la UE" (p. 224).

Los datos aportados en este trabajo, aunque con otra metodología y enfoque, corroboran los trabajos anteriores. Esto es así, al menos, en la red resultante que detecta los vínculos entre profesionales que trabajan en el mismo tipo de organizaciones.

Las razones de ello pueden obedecer a las motivaciones expuestas por diversos autores, entre ellos Kraut (Kraut, Galegher y Egido, 1988). La proximidad entre los investigadores, ayuda a la hora de determinar qué colaboradores son los adecuados, la definición de los problemas, a la 
hora de planificar los proyectos o de verificar la compatibilidad interpersonal e intelectual.

Como los contactos interpersonales son el fundamento de la colaboración, puede argumentarse que la proximidad geográfica entre los investigadores favorece la posibilidad de tener contactos o de asistir a cursos o acontecimientos profesionales en forma de conferencias o seminarios. La proximidad física crea oportunidades de comprobar si existen intereses comunes, de intercambiar ideas, de comprobar compatibilidades y de discutir las posibilidades de trabajar conjuntamente, todo ello en un marco de contactos cara a cara, que se ha demostrado como el preferido por los investigadores, y es por ello por lo que influye de forma manifiesta en la configuración de la red española.

Sin embargo, como se ha señalado por otros autores, los trabajos empíricos sobre coautoría, deben completarse con métodos cualitativos, si se desea obtener resultados integrados del fenómeno de la colaboración científica (Laudel, 2002).

\section{Conclusiones}

La finalidad de este trabajo ha sido la de describir y analizar los patrones de colaboración entre los profesores e investigadores que trabajan en las universidades españolas a partir de datos de coautoría de trabajos académicos. Los datos de tendencias desde el punto de vista del tipo de colaboración corroboran y confirman los referidos al conjunto de España (Moya Anegón et al., 2005).

Los datos se han presentado con una agregación por Comunidades Autónomas, en consonancia con otros trabajos que han caracterizado las regiones españoles con indicadores bibliométricos (Gómez, et al. 2005).

La red resultante del análisis de la colaboración entre pares que trabajan en organizaciones homogéneas y que desde el punto de vista institucional son muy próximas, parece estar presidido por un criterio de proximidad geográfica, con un fuerte componente regional que da origen a la constitución de subredes regionales de ámbito similar a la estructura organizativa territorial "federalizante" española.

Desde el punto de vista metodológico, puede afirmarse que la utilización de técnicas de visualización de redes sociales es idónea para representar gráficamente la configuración espacial de las redes resultantes de las actividades de coautoría.
Los resultados presentes sugieren otras líneas para la continuación de la investigación. En particular, aquellas que traten de responder a otros aspectos de la geografía de la investigación en colaboración entre investigadores procedentes de las universidades españolas; por ejemplo, las que analicen las redes en las que colaboran instituciones pertenecientes a otros sectores a nivel nacional, la obtención de redes institucionales por categorías temáticas o la elaboración de las que tengan por objeto la representación de la colaboración internacional.

\section{Referencias}

Batagelj, V.; Mrvar, A. (1997). Pajek. Program package for large networks analysis. // XVII Sunbelt Social Networks Conference San Diego, February,13-16 http://vlado.fmf.uni-lj.si/pub/networks/pajek/sunbelt.97/ pajek.htm (26-12-2007)

Beaver, D. D. (2004). Does collaborative research greater epistemic authority?. // Scientometrics. 60:3 (August 2004) 399-408.

Börner, K., Chen, C.; Boyack, K. (2003). Visualizing Knowledge Domains. // Annual Review of Information Science \& Technology. 37 (2003) 179-355.

Camí, J. (2004). Dinámica de intercitación y cocitación entre las universidades españolas: análisis bibliométrico de los documentos citados producidos en las universidades y de los documentos citantes en el período 1994-2000, a partir de las bases de datos National Citation Report del ISI. Madrid: Dirección General de Universidades, 2004.

Cruz Castro, L.; Sanz Menéndez, L.; Romero, M. (2004). Convergencia y divergencia en las políticas de ciencia y tecnología de los gobiernos regionales. Madrid:CSIC,2004. http://www.iesam.csic.es/doctrab2/dt0402.pdf

European Commission (2003). Third european report on science and technology indicators 2003: towards a knowledge-based economy. Luxembourg: Office for Official Publications of the European Communities, 2003.

Everett, M. G.; Borgatti, S.P. (1999) Peripheries of cohesive subsets. // Social Networks. 21:4 (October 2000) 397407.

Freeman, L. C. (2000). Visualizing Social Networks. // Journal of Social Structure. 1:1 (February 2000) 1-15.

Gómez, I.; et al. (2005) Regionalization of science and technology data in Spain. // Research Evaluation. 14:2 (August 2005) 137-148.

Gómez, I.; et al. (2007) Structure and research performance of spanish universities. Proceeedings of ISSI 2007: 11th International Conference of the International Society for Scientometrics and Informetrics. Madrid: CINDOC, 2007. 335-345.

Heffner, A. G. (1981). Funded research, multiple authorship, and sub-authorship collaboration in four disciplines. /I Scientometrics. 3:1 (January 1981) 5-12.

Kamada T.; Kawai S. (1989). An algorithm for drawing general undirected graphs. // Information Processing Letter. 31:1 (April 1989) 7-15.

Katz, J. S. (1994). Geographical proximity and scientific collaboration. // Scientometrics. 31:1 (September 1994) 31-43.

Kraut, R. E.; Galegher, J.; Egido, C. (1988). Relationships and task is scientific research collaboration. // HumanComputer interaction. 3:1 (1987-1988) 31:58. 
Leydesdorff, L.; Vaughan, L. (2006). Co-occurrence matrices and their applications in Information Science: Extending ACA to the Web Environment. // Journal of the American Society for Information Science \& Technology. 57:12 (October 2006) 1616-1628.

Laudel, G. (2002) What do we measure by co-authorships?. // Research Evaluation. 11:1 (April 2002) 3-15.

Liang, L.; Zhu, L. (2002). Major factors affecting China's inter-regional research collaboration: regional scientific productivity and geographical proximity. // Scientometrics. 55:2 (August 2002) 287-316.

Melin, G; Persson (1996). Studying collaboration using coauthorships. // Scientometrics. 36:3 (October 1996) 363377.

Moya Anegón, F.; et al. (2004). Análisis de la excelencia científica en la investigación universitaria española (1998-2002). Madrid: Dirección General de Universidades, 2004.

Moya Anegón F.; et. al. (2005). Indicadores bibliométricos de la actividad científica española (1990-2004). Madrid: Fundación Española para la Ciencia y la Tecnología, 2005.

Newman, M. E. J. (2001). The structure of scientific collaboration networks. // Proceedings of the National Academy of Sciences of the United States of America. 98:2 (January 2001) 404-409.

Okubo, Y.; Zitt, M. (2004). Searching for research integration across Europe: a closer look at international and interregional collaboration in France. // Science and Public Policy. 31:3 (June2004) 213-226.

Otte, E.; Rousseau, R. (2002). Social network anaysis: a powerful strategy, also for the information Sciences. // Journal of Information Science. 28:6 (December 2002) 441:453.

Persson, O.; Glänzel, W.; Danell, R. (2004). Inflationary bibliometric values: the role of scientific collaboration and the need for relative indicators in evaluative studies. // Scientometrics. 60:3 (August 2004) 421-432.

Rovira Pato, LI. (2006). Análisis de la actividad interdisciplinar en base a los documentos ISI de las universidades españolas: estudio de las aplicaciones para su óptima evaluación. Madrid: Dirección General de Universidades, 2006.

Sanz Menéndez, L. (1997). Estado, ciencia y tecnología en España: 1939-1997. Madrid: Alianza Universidad, 1997.

Smith, D.; Katz, J. S. (2002). Collaborative approaches to research. HEFCE fundamental review of research policy and funding. A joint project with the Higher Education Policy Unit (HEPU), University of Leeds and the Science Policy Research Unit (SPRU) University of Sussex. http://www.sussex.ac.uk/Users/sylvank/pubs/collc.pdf (2008-05-14).

Vargas-Quesada, B.; Moya Anegón, F. (2007). Visualizing the structure of science. Berlin: Springer, 2007.

Tichy, N. M.; Tushman, M. L.; Fombrun, Ch. (1979). Social network analysis for organization. // The Academy of Management Review. 4:4 (October 1979) 507-519.

Wagner, C. S.; Leydesdorff, L. (2005). Network, selforganization and the growth of international collaboration in science. // Research Policy. 34:10, (December 2005) 1608-1618.

Zitt, M.; Bassecoulard, E.; Okubo, Y. (2000). Shadows of the past in international cooperation. Collaboration profiles of the top five producers of science. // Scientometrics. 47:3 (March 2000) 627-657. 